\title{
TIGHT AND TAUT EMBEDDINGS AND PROJECTIVE TRANSFORMATION
}

\author{
BY C.-S. CHEN
}

Communicated by S. S. Chern, April 21, 1977

Let $f: M^{n} \rightarrow R^{N}$ be an embedding of an $n$-dimensional compact differentiable manifold $M$ into a euclidean vector space $R^{N}$. Any hyperplane in $R^{N}$ has an equation $z=c$, where $z$ is a linear function and $c$ a real number. For the oriented hyperplane $z=c$ we use the notation ( $z f=z \circ f$ is the composition)

$$
(z f)_{c}^{-}=\{x \in M: z f(x)<c\}
$$

to indicate the image of $f$ on the negative side of the hyperplane. The closure of $(z f)_{c}^{-}$is

$$
\left(z f^{\prime}\right)_{c}=\{x \in M: z f(x) \leqslant c\} .
$$

The embedding $f$ is called tight if for almost all $z$ and $c$ the inclusion $i:(z f)_{c} \rightarrow$ $M$ induces a monomorphism $i_{*}: H_{*}\left((z f)_{c}, F\right) \rightarrow H_{*}(M, F)$ of homology groups, where the coefficient $F$ is chosen to satisfy condition 3A of [5] (or (3) of [6]).

Besides the collection of hyperplanes, other interesting geometric objects are hyperspheres. A hypersphere of $R^{N}$ is given by an equation $d_{p}(x)=c$, where $d_{p}(x)$ is the distance from $x$ to a fixed point $p$ of $R^{N}$ and $c$ is a positive real number. The hypersphere is naturally oriented so that its inner part is the positive side of the hypersphere. We define analogously the sets $\left(d_{p} f\right)_{c}^{-}$and $\left(d_{p} f\right)_{c}$. The embedding $f$ is called taut if for almost all $p$ and $c>0$ the inclusion $i$ : $\left(d_{p} f\right)_{c} \rightarrow M$ induces a monomorphism $i_{*}: H_{*}\left(\left(d_{p} f\right)_{c}\right) \rightarrow H_{*}(M)$ where the coefficient is chosen to be the same as before and hence is omitted. Originally both concepts, tight and taut, are defined in terms of the Morse height functions and Morse distance functions respectively, and this is why in both definitions we use "almost all". It seems that the definition with "almost all" should imply the one without "almost all". The answer for this is still unknown. However, for the special class of embeddings we are interested in, the definition with "almost all" is equivalent to the one without "almost all".

The geometrical content of an embedding $f: M^{n} \rightarrow R^{N}$ is given by a symmetric tensor $T$ defined as follows: For any two tangent vectors $x, y$ of $M$ at $p$ $\in M$ we first extend $x, y$ to vector fields $X, Y$ respectively near $p$. We define

AMS (MOS) subject classifications (1970). Primary 53A05, 53A20, 53C99.

Key words and phrases. Tight, taut, projective transformation, extreme, second fundamental form, Morse theory. 


$$
\begin{aligned}
T_{x} y= & \text { normal component of } \bar{\nabla}_{x} y \text { with respect to the } \\
& \text { tangent space of } M \text { at } p \text {, where } \bar{\nabla} \text { is the covari- } \\
& \text { ant differentiation in } R^{N} .
\end{aligned}
$$

The embedding $f$ is said to be convex if $x \neq 0$ implies $T_{x} x \neq 0$. An ovoid is a closed convex hypersurface. An embedding with image lying on an ovoid will be called an extreme embedding.

The third concept we need is "projective transformation". Given an embed$\operatorname{ding} f: M^{n} \rightarrow R^{N}$ of a compact $M^{n}$ into $R^{N}, R^{N}$ can be considered as an affine $N$-space $A^{N}$ and can be closed by adding the class of parallel lines as new points. So we obtain in this way a natural embedding $i: A^{N} \rightarrow P^{N}$ of $A^{N}$ onto the complement of a hyperplane $P^{N-1}$ in the real projective $N$-space $P^{N}$. Following Kuiper [5], the map $f$ is called projectively equivalent to another $f^{\prime}: M^{n} \rightarrow \bar{R}^{N}$ if $\bar{R}^{N}$ is obtained from $P^{N}$ by a different choice of $P^{N-1}$ which does not meet the image of $M$ in $P^{N}$. An embedding $f$ is said to be substantial if the image is not contained in a hyperplane.

THEOREM. Let $f$ be a substantial tight extreme embedding of $S^{m} \times S^{n}$ into $R^{m+n+2}$ where $m / n \neq 2,1 / 2$. Then $f$ is projectively equivalent to a product embedding of two ovoids: $f=f_{1} \times f_{2}$ where $f_{1}: S^{m} \rightarrow R^{m+1}$ and $f_{2}: S^{n} \rightarrow$ $R^{n+1}$ are two ovoids.

This theorem is related to the theorem of Chern and Lashof $[4$, Theorem 4]. By composing with a stereographic projection, we can bring a hypersurface into a codimension-two extreme embedding.

Corollary. Let $f$ be a substantial taut embedding of $S^{m} \times S^{n}$ into $R^{m+n+1}$. Then $f$ is related by stereographic projection to a product embedding.

This corollary generalizes Banchoff's theorem of STPP [1, Theorem 5.5].

The first step of the proof of the theorem is to modify Lemma 2.1 of [2]. Instead of arguing with determinants we use a restriction on indices of the second fundamental form. This restriction is inherited through Morse theory from the assumption that the embedded manifold is a topological $(m, n)$-torus. $\mathbf{A}$ slightly different method enables us to prove Theorem $C$ of [2]. In [3], we use the curvature assumption to prove the product is an orthogonal product. Here we prove the product is orthogonal after a proper projective transformation. The projective transformation is found by using the concept of dual manifold. The study of the dual manifold is the second step of the proof of the theorem. Details will appear elsewhere.

\section{REFERENCES}

1. T. F. Banchoff, The spherical two-piece property and tight surfaces in spheres, J. Differential Geometry 4 (1970), 193-205. MR 42 \#3720.

2. C.-S. Chen, On tight isometric immersion of codimension two, Amer. J. Math. 94 (1972), 974-990. MR 51 \#11364. 
3. C.-S. Chen, More on tight isometric immersion of codimension two, Proc. Amer. Math. Soc. 40 (1973), 545-553. MR 51 \#11365.

4. S. S. Chern and R. K. Lashof, On the total curvature of immersed manifolds, Amer. J. Math. 79 (1957), 306-318. MR 18, 927.

5. N. H. Kuiper, On convex maps, Nieuw Arch. Wisk. 10 (1962), 147-164. MR 26 \#3076.

6. Immersions with minimal absolute total curvature, Invent. Math. 10 (1970) 209-238.

7. Tight topological embeddings of the Moebius band, J. Differential Geometry 6 (1972), 271-283. MR 47 \#2609.

SCHOOL OF MATHEMATICS, UNIVERSITY OF MINNESOTA, MINNEAPOLIS, MINNESOTA 55455 